\title{
Screening for implicated genes in colorectal cancer using whole-genome gene expression profiling
}

\author{
LONG-CI SUN $^{1,2}$ and HAI-XIN QIAN ${ }^{1}$ \\ ${ }^{1}$ Department of General Surgery, The First Affiliated Hospital of Soochow University, Suzhou, Jiangsu 215006; \\ ${ }^{2}$ Department of Gastrointestinal Surgery, Renji Hospital, School of Medicine, Shanghai Jiao Tong University, \\ Shanghai 200127, P.R. China
}

Received October 24, 2017; Accepted March 2, 2018

DOI: $10.3892 / \mathrm{mmr} .2018 .8862$

\begin{abstract}
To identify biologically relevant genes associated with the pathogenesis of colorectal cancer (CRC), genome wide expression profiles of 17 pairs of CRC tumor and adjacent tissues, previously published in a DNA microarray study, were analyzed. Cytoscape, String tools and DAVID tools were used to investigate the biological pathways encoded by the genes identified as being either upregulated or downregulated in $\mathrm{CRC}$, to determine protein-protein interactions and to identify potential hub genes associated with CRC. As a result, a total of 3,264 genes were identified as being differentially expressed in CRC and adjacent tissues, including 1,594 downregulated and 1,670 upregulated genes. Furthermore, 306 genes were revealed to be clustered in a complex interaction network, and the top 20 hub genes in this network were determined by application of the Matthews Correlation Coefficient algorithm. In addition, the patterns of the expression levels of the 20 hub genes were investigated using reverse transcription-quantitative polymerase chain reaction. Gene Ontology analysis revealed that four of the 20 hub genes encoded small subunit processome components (UTP3 small subunit processome component; UTP14 small subunit processome component; UTP 18 small subunit processome component; and UTP20 small subunit processome component) and a further four encoded WD repeat domains (WD repeat-containing protein 3, WD repeat domain 12, WD repeat-containing protein 43 and WD repeat-containing protein 75 ). In conclusion, the present DNA microarray study identified genes involved in the pathogenesis of CRC. Furthermore, it was revealed that hub genes identified from among the total identified upregulated and downregulated genes in CRC encoding subunit processome components and
\end{abstract}

Correspondence to: Professor Hai-Xin Qian, Department of General Surgery, The First Affiliated Hospital of Soochow University, 899 Pinghai Road, Suzhou, Jiangsu 215006, P.R. China E-mail: qianhaixin1@hotmail.com

Key words: colorectal cancer, microarray, hub gene, expression profiling
WD repeat domains may represent novel target molecules for future treatments of CRC.

\section{Introduction}

Colorectal cancer (CRC) is one of the most commonly diagnosed cancers worldwide (1). It is the second most prevalent cancer type among women, following breast cancer, and the third most prevalent cancer type among men $(2,3)$. In recent decades, diagnoses and treatment of CRC have significantly improved. However, the disease remains a major public health problem worldwide. In 2016, the American Cancer Society estimated 70,820 new male cases and 63,670 new female cases of CRC would be diagnosed, and that there would be 49,190 CRC associated deaths in the USA (4). The high rate of mortality of the disease is in part due to limitations in the currently available therapies for CRC treatment, which is the result of an incomplete understanding of the biological mechanisms underlying the disease.

It has been well established that genes have an important role in tumor pathogenesis, and that the transcriptional activation or inhibition of certain genes serves an important role in the development and progression of the majority of human tumors (5). The analysis of genome-wide gene expression levels via DNA microarray experiments is a recently developed systematic approach that is used to gain comprehensive information regarding tumor associated gene transcription profiles (6). This information can lead to the identification of prognostic biomarkers $(7,8)$, enable the discrimination between various histological subtypes of tumors $(9,10)$.

Interactions between genes can affect the pathogenesis of CRC and tumor progression. The aim of the present study was to identify biologically relevant genes that may be associated with the pathogenesis of CRC, via analysis of genome-wide expression profiles in CRC. Following the identification of differentially expressed genes (DEGs), the present study further aimed to determine the hub genes involved in the regulatory pathways of CRC progression, by Gene Ontology (GO) analysis, and to subsequently derive a gene interaction network. Identification of hub genes in CRC and the development of a gene interaction network may reveal the biological characteristics of CRC tumor development and enable the development of novel molecular-targeted therapies for the treatment of CRC. 


\section{Materials and methods}

Data collection. The gene expression profiling dataset from previously published relevant study was retrieved from the NCBI GEO database (accession number GSE32323; www.ncbi.nlm.nih.gov/geo) (11). Data regarding a total of 17 paired samples of CRC tumors and adjacent tissues were available from the GSE32323 dataset.

Data processing. Raw RNA expression profile datasets were preprocessed using $\mathrm{R} 3.4 .1$ statistical software (https://www.r-project.org/) together with Affy package (12). In accordance with the R-package, the gcrma algorithm (13) was used to adjust for background intensities in the Affymtrix array data by including optical noise and non-specific binding. The background adjusted probe intensities were then converted into expression measures using the normalization and summarization methods included in the multiarray average algorithm (12). The k-Nearest Neighbor algorithm (14) was subsequently used to generate the missing values.

Microarray and statistical analysis. The R-package and limma (www.bioconductor.org.uk) (15) were used to investigate gene expression microarray data. Limma uses linear models, as well as empirical Bayesian methods, to investigate the differential expression levels in the GSE32323 dataset. Fold-change (FC) values were calculated using the ratio of the geometric means of the gene expression levels between the two treatment groups (CRC tissue vs. adjacent tissue). A Wilcoxon Rank-Sum Test was performed to determine the statistical significance of differences between the groups $(\mathrm{P}<0.05$ was considered statistically significant). Genes were selected for if the $\log 2-\mathrm{FC}$ values were $>1$ (equivalent to a FC value of $>2.0$ or $<0.5$ ), and P-values determined by the Wilcoxon Rank-Sum Test were $\mathrm{P}<0.0002$. Hierarchical clustering of the selected genes was performed with $\mathrm{R}$ software using the Euclidean distance and complete linkage method. Regarding clustering, the expression data were standardized via conversion to $\mathrm{z}$ scores (mean $=0$, variance $=1$ ) for each probe. Following this, protein-protein interactions among the selected genes were analyzed using the reference data contained in the String database of known protein-protein interactions, version 10.5 (string-db.org/). Furthermore, the weight of each edge of the gene clusters was calculated using molecular complex detection (MCODE) software (version 1.4.2) from Cytoscape, version 3.5.1 (apps.cytoscape.org/apps/mcode) (16). In addition, the Cytoscape (www.cytoscape.org/) plug-in CytoHubba, version 0.1 (http://apps.cytoscape.org/apps/cytohubba) was used to identify hub genes among the selected genes. Finally, BiNGO software version 3.0.3 (http://apps.cytoscape.org/ apps/bingo) from Cytoscape was used to determine which GO categories represented identified genes, and Kyoto Encyclopedia of Genes and Genomes (KEGG) pathways (http://www.genome.jp/kegg/) were analyzed using DAVID functional annotation tools (version 6.8) $(17,18)$.

Collection of CRC samples. The present study also investigated 3 patients ( 2 males and 1 female; aged 48-56 years old) with CRC who had previously undergone radical resection of CRC in the Gastroenterology Department of Renji
Hospital Affiliated to Shanghai Jiaotong University School of Medicine (Shanghai, China) between November 2017 and December 2017. The inclusion criteria for patients were as follows: i) colorectal adenocarcinoma confirmed by pathological examination; ii) the patients must have not received a radical resection prior and must not have been treated with preoperative chemoradiotherapy. Characteristics of patients included in the present study are presented in Table I. Written informed consent was obtained from all patients included in the present study. The Ethics Committee of Renji Hospital Affiliated to Shanghai Jiaotong University School of Medicine approved the present study and protocols. CRC tissue samples (diameter of $0.5 \mathrm{~cm}$ ) were obtained via tumor dissection. Paracancerous tissue (distal tissue $3 \mathrm{~cm}$ away from the tumor) was also obtained from patients via dissection. Paracancerous tissues and cancerous tissues were collected as normal tissues and tumor tissues, respectively. RNA was extracted from these tissues by TRIzol (Invitrogen; Thermo Fisher Scientific, Inc., Waltham, MA, USA) for future experiments.

Reverse transcription-quantitative polymerase chain reaction $(R T-q P C R)$. In order to further investigate the results of the microarray analysis, the 20 hub genes identified to be associated with CRC were subjected to RT-qPCR analysis. A total of $1 \mu \mathrm{g}$ of RNA from tumor or normal tissue samples was reverse transcribed to cDNA using SuperScript ${ }^{\mathrm{TM}}$ II reverse transcriptase (Invitrogen; Thermo Fisher Scientific, Inc.; heat mixture to $65^{\circ} \mathrm{C}$ for $5 \mathrm{~min}$, incubate at $42^{\circ} \mathrm{C}$ for $50 \mathrm{~min}$, inactivate the reaction by heating at $70^{\circ} \mathrm{C}$ for $15 \mathrm{~min}$. RT-qPCR was performed using cDNA, $\mathrm{iQ}^{\mathrm{TM}} \mathrm{SYBR}$ Green Supermix (Invitrogen; Thermo Fisher Scientific, Inc.) and primers $(2.5 \mu \mathrm{M})$. The reaction was performed using the Bio-Rad RT-qPCR detection system (Bio-Rad Laboratories, Inc., Hercules, CA, USA) under the following PCR conditions: Initial denaturation at $95^{\circ} \mathrm{C}$ for $5 \mathrm{~min} ; 35$ cycles at $95^{\circ} \mathrm{C}$ for $30 \mathrm{sec}$; $60^{\circ} \mathrm{C}$ for $30 \mathrm{sec} ; 72^{\circ} \mathrm{C}$ for $30 \mathrm{sec}$; and final extension at $72^{\circ} \mathrm{C}$ for $5 \mathrm{~min}$. Expression levels of these genes were normalized to the house-keeping gene, $\beta$-actin via the $2^{-\Delta \Delta \mathrm{Cq}}$ method (19). Primer sequences used were as follows: WD repeat-containing protein 3 (WDR3) forward, 5'-AATCCAGCGGGTGAC TAA-3' and reverse, 5'-ACAGGCTGAGGAGTAGGC-3'; WD repeat-containing protein 43 (WDR43) forward, 5'-TGACTT ATTGGCTCTTGG-3' and reverse, 5'-GGCTGATACATA GGGAAC-3'; UTP14 small subunit processome component (UTP14A) forward, 5'-GCTGTGGAGGCGAGTAAG-3', and reverse, 5'-GCCAATGGTGGGTAAATG-3'; damage specific DNA binding protein 1 and cullin4 associated factor 13 (DCAF13) forward, 5'-GGGATGAACAAAGAACTAA-3' and reverse, 5'-AGATTTATCGAAACTAGCAG-3'; KRR1 small subunit processome component (KRR1) forward, 5'-GGCAGC ATGACTGTTTGT-3' and reverse, 5'-TAAGCCGTTGTCTTC GTT-3'; digestion organ expression factor (DIEXF) forward, 5'-GAGAAGCATCCGACTCTA-3', and reverse, 5'-TTAAAC CTGGCATCAATC-3'; HEAT repeat containing 1 (HEATR1) forward, 5'-ATTCACTTGTCGCCTTAC-3', and reverse, 5'-TCTTGTCTCGTGGTATGG-3'; WD repeat-containing protein 75 (WDR75) forward, 5'-ATGCATTGCGAATATCCA AAAGAGC-3', and reverse, 5'-GCTCTTTTGGATATTCGC AATGCAT-3'; UTP 18 small subunit processome component (UTP18) forward, 5'-GTTTATGTTTGGGATGTGA-3', and 
Table I. Characteristics of patients with colorectal cancer in the present study.

\begin{tabular}{lllcll}
\hline Patient & Age & Sex & Stage & Preoperative chemoradiotherapy & Surgical resection \\
\hline 1 & 48 & Male & $2 \mathrm{a}$ & No & Radical correction \\
2 & 53 & Male & $3 \mathrm{c}$ & No & Radical correction \\
3 & 56 & Female & $2 \mathrm{~b}$ & No & Radical correction \\
\hline
\end{tabular}

reverse, 5'-GGCTTTGGGTTTGTTTCT-3'; UTP 3 small subunit processome component (UTP3) forward, 5'-AATGCC GATGATGATGGT-3' and reverse, 5'-CAAGTATTGGCT TCCTTTT-3'; RNA terminal phosphate cyclase-like protein (RCL1) forward, 5'-TGGAGGAAATCTACAGGG-3' and reverse, 5'-CACTTGAGGGTCTTGCTAA-3'; small subunit processome complement 20 (UTP20) forward, 5'-AGACCG AGAACACCTACC-3' and reverse, 5'-CAACCTCCTCCTCAT AGC-3'; testis-expressed protein 10 (TEX10) forward, 5'-ATG TCGAGAATGACTAAAAAAAGAA-3' and reverse, 5'-TTC TTTTTTTAGTCATTCTCGACAT-3'; WD repeat domain 12 (WDR12) forward, 5'-ACATACTGGTTGGGTGAC-3' and reverse, 5'-ATGGGAAGTGGTAGGTGA-3'; exosome component 5 (EXOSC5) forward, 5'-GAGGAGACGCATACT GACGC-3' and reverse, 5'-ACACCAGGCAGCCCAATC-3'; neutral invertase (regulatory particle non-ATPase 12) binding protein 1 (NOB1) forward, 5'-TGTGAGCCTGAGAACCTG G-3' and reverse, 5'-CTGCTGGATCTGCTTGATGT-3'; SDA1 domain containing 1 (SDAD1) forward, 5'-TGCCGCAGTTAC AGAATC-3', and reverse, 5'-GTGCCATAAACATCACCA G-3'; DEAD-box helicase 27 (DDX27) forward, 5'-GCCCGT GGACTTGACATT-3' and reverse, 5'-GCCGCTTTGCTGTAT TGA-3'; ribosome production factor 2 (RPF2) forward, 5'-TCC GCCTGGCTGGATTAG-3' and reverse, 5'-TTCCTGGTTTGT AGTTTGCTTA-3'; GTP binding protein 4 (GTPBP4) forward, 5'-CTAAAGATTATGTGCGACTG-3', and reverse, 5'-ATG GTGTTCCTATCCTCC-3'; and $\beta$-actin forward, 5'-CTCCAT CCTGGCCTCGCTGT-3' and reverse, 5'-GCTGTCACCTTC ACCGTTCC-3'.

Statistical analysis. SPSS software (version 22.0; IBM Corp., Armonk, NY, USA) was used to perform statistical analysis. Expression levels of genes in paracancerous tissues and cancerous tissues were compared using two-tailed Student's t-tests. The bar plot was generated by GraphPad Prism (version 6.0; GraphPad Software, Inc., La Jolla, CA, USA). The data in the present study was presented as the mean \pm standard deviation. $\mathrm{P}<0.05$ was considered to indicate a statistically significant difference.

\section{Results}

Characterization of differentially expressed genes. A total of 3,264 genes were identified by the Affymetrix Human Genome Array analysis as being differentially expressed in CRC tissues compared with adjacent tissue $(\mathrm{P}<0.05$; data not shown). This included 1,594 genes that were downregulated $(\mathrm{FC}<0.5$ in expression in CRC vs. adjacent tissue) and 1,670 genes that were upregulated (FC>2.0 in expression in CRC vs. adjacent tissue) compared with the adjacent tissue. The former group was defined as the 'lower group' and the latter group as the 'higher group'. GO and KEGG enrichment analyses were then performed to identify the biological characteristics of each of these gene subgroups.

In the lower group, a total of 1,594 downregulated genes were revealed to be significantly enriched in 75 KEGG pathways, the top 20 of which are presented in Fig. 1A $(\mathrm{P}<0.05)$. The protein kinase, guanosine monophosphate-protein kinase G signaling pathway (hsa04022) was the most notably enriched pathway in the lower group (Fig. 1A). In addition, numerous well-established signaling pathways were also enriched, including the mitogen-activated kinase-like protein signaling pathway (hsa04010), cyclic adenosine monophosphate signaling pathway (hsa04024) and the phosphoinositide 3 kinase-protein kinase B signaling pathway (hsa04151). Furthermore, pathways associated with melanoma (hsa05218) and bladder cancer (hsa05219) were also enriched in CRC, with 10 and 7 genes identified as being associated with these diseases, respectively. In addition, the results of GO classification of the identified genes into the categories 'biological process', 'molecular function' and 'cellular component', as performed by BiNGO from Cytoscape, revealed that in the 'biological process' category, the genes were notably enriched in the following GO terms: 'Positive regulation of IкB kinase/nuclear factor- $\kappa$ B signaling' (GO: 0043123), 'negative regulation of cell growth' (GO: 0030308) and 'negative regulation of growth' (GO: 0045926; Fig. 1B). GO terms associated with the identified enriched genes belonging to the 'cellular component' and 'molecular function' categories are presented in Fig. 1C and D, respectively.

In the higher group, a total of 1,670 upregulated genes were revealed to be significantly enriched in 28 KEGG signaling pathways, the top 20 genes of which are presented in Fig. 2A. The 'cell cycle' (hsa04110) pathway represents the most enriched pathway within this group, followed by the 'cellular tumor antigen p53 signaling pathway' (hsa04115) and the 'Wnt protein signaling pathway' (hsa04310). In the 'biological process' GO category, genes were notably enriched in the following GO terms: 'Mitotic nuclear division' (GO: 0007067), 'DNA replication' (GO: 0006260), 'sister chromatid cohesion' (GO: 0007062) and ' $\mathrm{G} 1 / \mathrm{S}$ transition of mitotic cell cycle' (GO: 0000082; Fig. 2B). In the 'cellular component' category, genes were significantly enriched in the GO term associated with the 'nucleoplasm' (GO: 0005654; Fig. 2C), and in the 'molecular function' category, the genes were most significantly enriched in GO terms associated with 'protein binding' (GO: 0005515) and 'poly (A) RNA binding' (GO: 0044822; Fig. 2D).

Analysis of protein-protein interactions of the differentially expressed genes. To determine the protein-protein interactions 
Downregulation genes

A

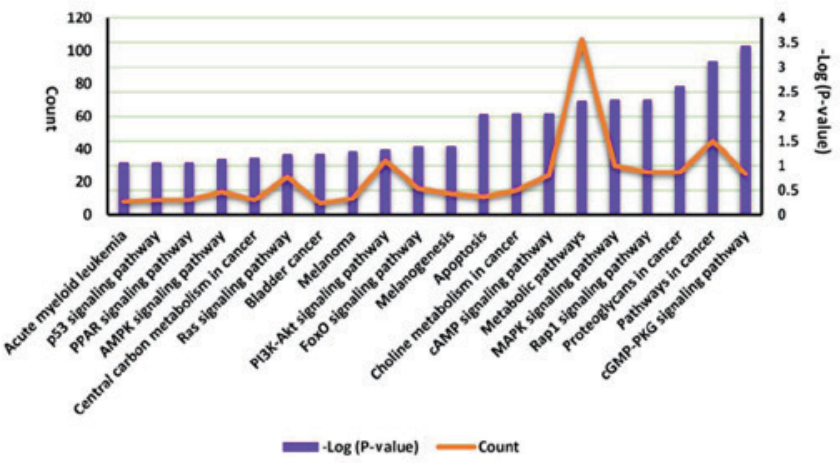

B

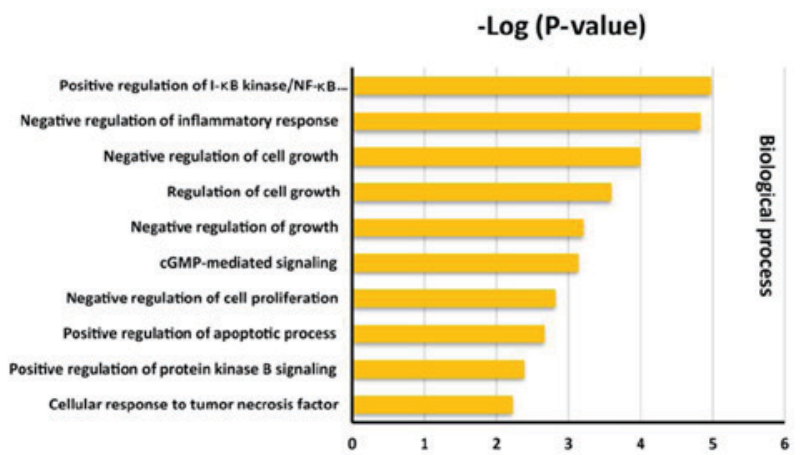

$c$

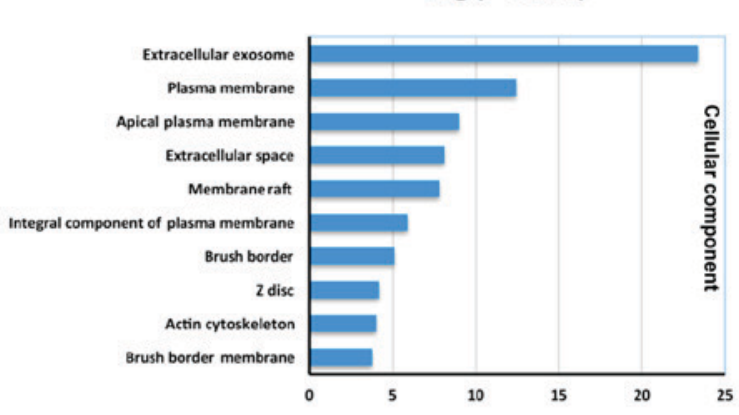

D

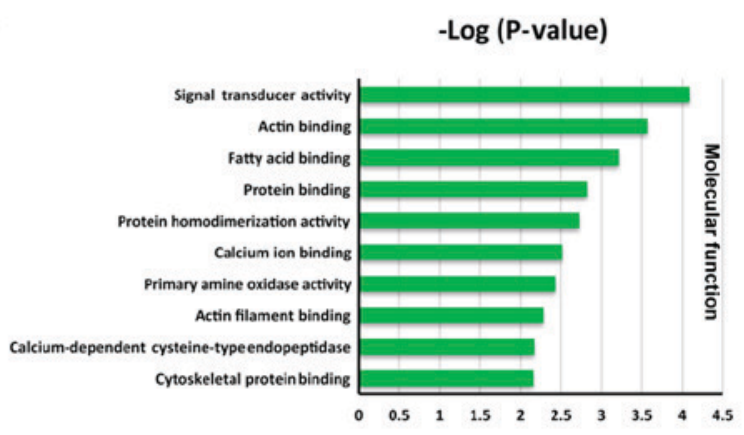

Figure 1. Enrichment analysis of the 1,594 downregulated genes. (A) The top 20 enriched pathways (purple columns) in the Kyoto Encyclopedia of Genes and Genomes database and the gene count (orange line) enriched in each pathway are presented. GO enrichment analysis revealing the top $10 \mathrm{GO}$ terms associated with (B) 'biological process', (C) 'cellular component' and (D) 'molecular function' are presented. GO, Gene Ontology; p53, cellular tumor antigen p53; PPAR, peroxisome proliferator-activated receptor; AMPK, AMP protein kinase; PI3K-Akt, phosphoinositide 3-kinase-protein kinase B; FoxO, forkhead box sub-group O; cAMP, cyclic adenosine monophosphate; MAPK, mitogen-activated protein kinase; Rap1, Rab5 activating protein 1; cGMP-PKG, guanosine monophosphate-protein kinase $\mathrm{G}$.

of the selected genes, the String tool was used. The results revealed that 306 of the genes were clustered in a complex interaction network (Fig. 3A), thus suggesting close interactions between the proteins encoded by such genes. Furthermore, calculation of the weighted index between each encoded protein revealed the existence of three subgroups within the larger network, which were closely associated with each other (subgroups are represented in blue, green and light blue shading in Fig. 3A). Each subgroup was extracted and their interactions are separately presented in Fig. 3B.

Identification of hub genes for CRC. To identify potential hub genes among the 306 genes previously identified, Matthew's correlation coefficient algorithms were performed using the CytoHubba software plug-in. As presented in Fig. 4, 20 potential hub genes for CRC were identified, all of which were closely associated with each other. The characteristics of the 20 hub genes are presented in Table II. Four of the 20 hub genes are genes that encode for small subunit processome components (UTP3, UTP14A, UTP18 and UTP20) and four are genes that encode for WD-repeat domains (WDR3, WDR12, WDR43, WDR75). The remaining 12 genes encode binding domains or proteins.

Verification of Affymetrix gene expression data. To further investigate the gene expression profiles, the expression levels of the 20 hub genes were determined via RT-qPCR analysis. The results demonstrated that the majority of the expression levels of the 20 hub genes were significantly upregulated in CRC tissues compared with control tissues (Fig. 5), which is consistent with the results of the microarray data analyses.

\section{Discussion}

$\mathrm{CRC}$ progression is characterized by the transformation of the normal mucosa of the bowel into an adenoma and then into a malignant tumor. This progression involves the acquisition of gene mutations that enable the tumor cells to proliferate and migrate within the tissues (20).

In the present study, a previously published microarray dataset (11) was used to identify significant genes in CRC tumor tissues via comparison of gene expression profiles in CRC tissues with those in adjacent paired tissue samples. A total of 1,594 genes were revealed to be downregulated, and 1,670 genes were revealed to be upregulated, in CRC tumor tissues. KEGG enrichment analysis of each of these subgroups provided information regarding the function and other biological characteristics of associated genes. The upregulated genes were predominantly enriched in 'cell cycle' (hsa04110), 'RNA transport' (hsa03013) and 'DNA replication' (hsa03030) pathways, which is consistent with the findings of a previous study investigating gene involvement in 

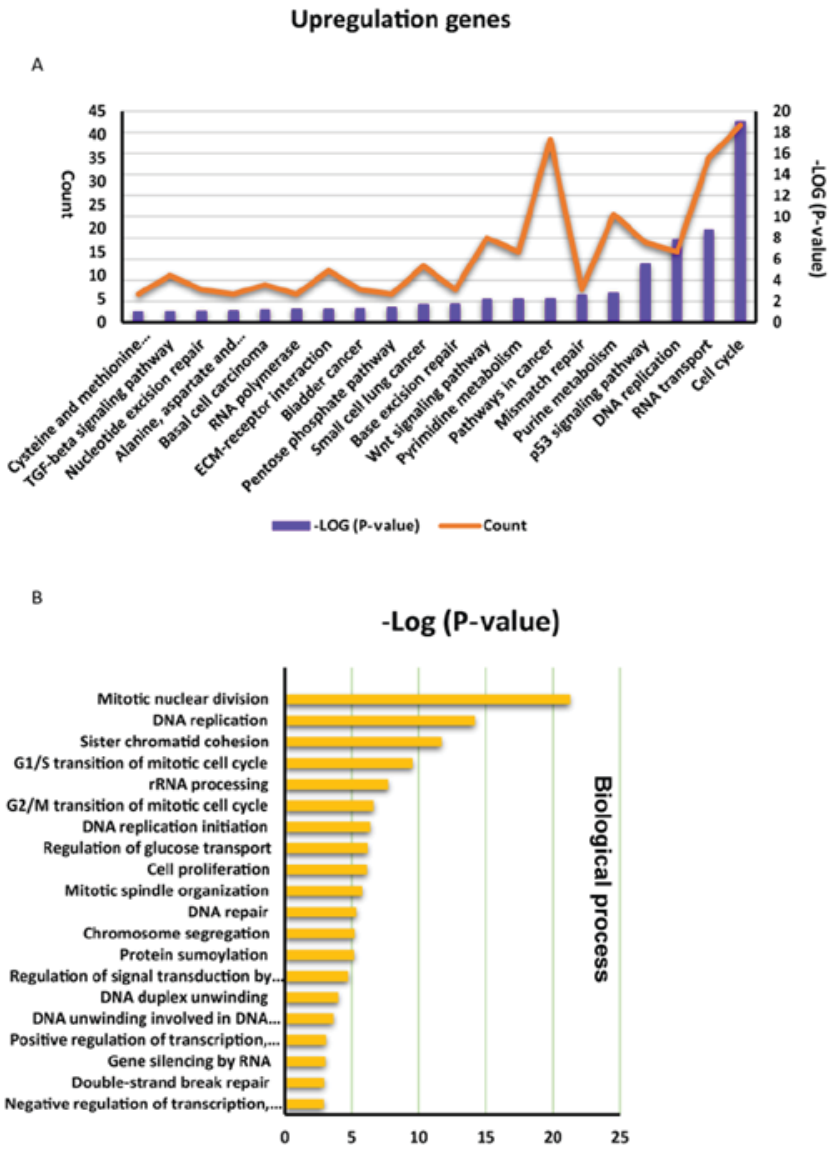

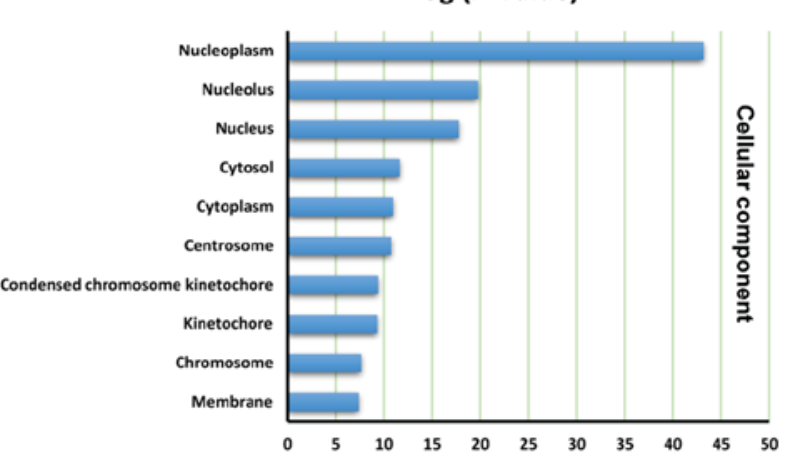

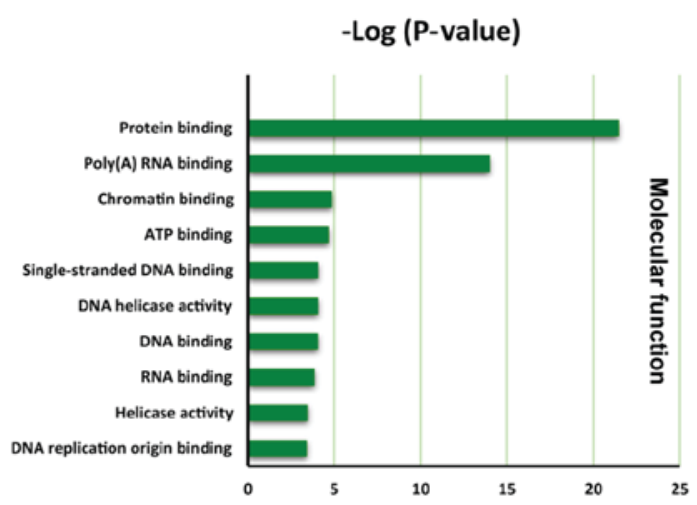

Figure 2. Enrichment analysis of the 1,670 upregulated genes in colorectal cancer. (A) The top 20 enriched pathways (purple columns) in the Kyoto Encyclopedia of Genes and Genomes database and the gene count (orange line) enriched in each pathway are presented. GO enrichment analysis revealing the top 10 GO terms associated with (B) 'biological process', (C) 'cellular component' and (D) 'molecular function' are presented. GO, Gene Ontology; p53, cellular tumor antigen p53; TGF, tumor growth factor; ECM, extracellular matrix; rRNA, ribosomal RNA.

tumor pathogenesis (21). The mammalian cell cycle is highly organized and regulated to ensure the correct functioning of cell division and other biological activities (22). The four phases of the cell cycle $(\mathrm{G} 0 / \mathrm{G} 1, \mathrm{~S}, \mathrm{G} 2$ and $\mathrm{M})$, are regulated by numerous cyclin-dependent kinases (CDKs) (23). Aberrant cell cycle activity, which may occur as a result of genetic lesion within genes encoding cell cycle proteins, represents one of the typical characteristics of cancer (24). Therefore, synthetic inhibitors of CDKs are widely used as anticancer drugs in current cancer treatment therapies $(25,26)$.

Notably, in the present study, the upregulated genes identified in CRC were also revealed to be enriched in 'small cell lung cancer' (hsa05222), 'bladder cancer' (hsa05219) and 'basal cell carcinoma' (hsa05217) KEGG pathways, suggesting that CRC may exhibit similar gene regulation and molecular mechanisms with small cell lung cancer, bladder cancer and basal cell carcinoma. Similarly, the results from the enrichment analysis of the downregulated genes revealed that there were 10 and 7 downregulated DEGs enriched in the 'melanoma' (hsa05218) term and 'bladder cancer' (hsa05219) term, respectively. Merlo et al (27) revealed that one of the microsatellite alterations exhibited in small cell lung cancer is also exhibited in CRC. Furthermore, a clinical study in Japan has suggested that patients with bladder cancer have the potential to develop colon cancer $(1.44 \%)$ during anticancer therapy (28), and an immunotherapy study have demonstrated that type I and II interferons can be used to treat both CRC and melanoma due to their dual role in promoting proliferation and inhibiting growth (29). However, the extent to which genes that have been previously revealed to be implicated in these other cancers are expressed in CRC remains largely unknown, and warrants further research.

Song et al (30) downloaded CRC microarray data from the GEO database (GSE17538) and screened for genes associated with enhancer of zeste homolog 2 (EZH2). Song et al (30) revealed that EZH2 may represent a potential prognostic marker of patients with CRC. By contrast, the present study was performed using all DEGs in CRC, and aimed to uncover the molecular mechanisms underlying the progression of CRC. Liang et al (31) analyzed 141 samples (132 CRC and 9 normal colon epitheliums) and 3,500 DEGs were identified. In addition, Liang et al (31) performed GO and KEGG pathway enrichment analyses, and the top 10 hub genes from PPI network were identified, including $G$ protein subunit $\gamma 2$, angiotensin precursor, serum amyloid A1, adenylate cyclase 5, lysophosphatidic acid receptor 1 , neuromedin- $\mathrm{U}$, interleukin 8 , C-X-C motif chemokine ligand $12, \mathrm{G}$ protein subunit $\alpha 1$, and $\mathrm{C}-\mathrm{C}$ motif chemokine receptor 2 . There was no intersection between the 10 hub genes and 20 hub genes detected in the present study, which suggested that the novel CRC-associated genes were identified. Furthermore, Guo et al (32) acquired overlapping DEGs in CRC from four GEO datasets 
A

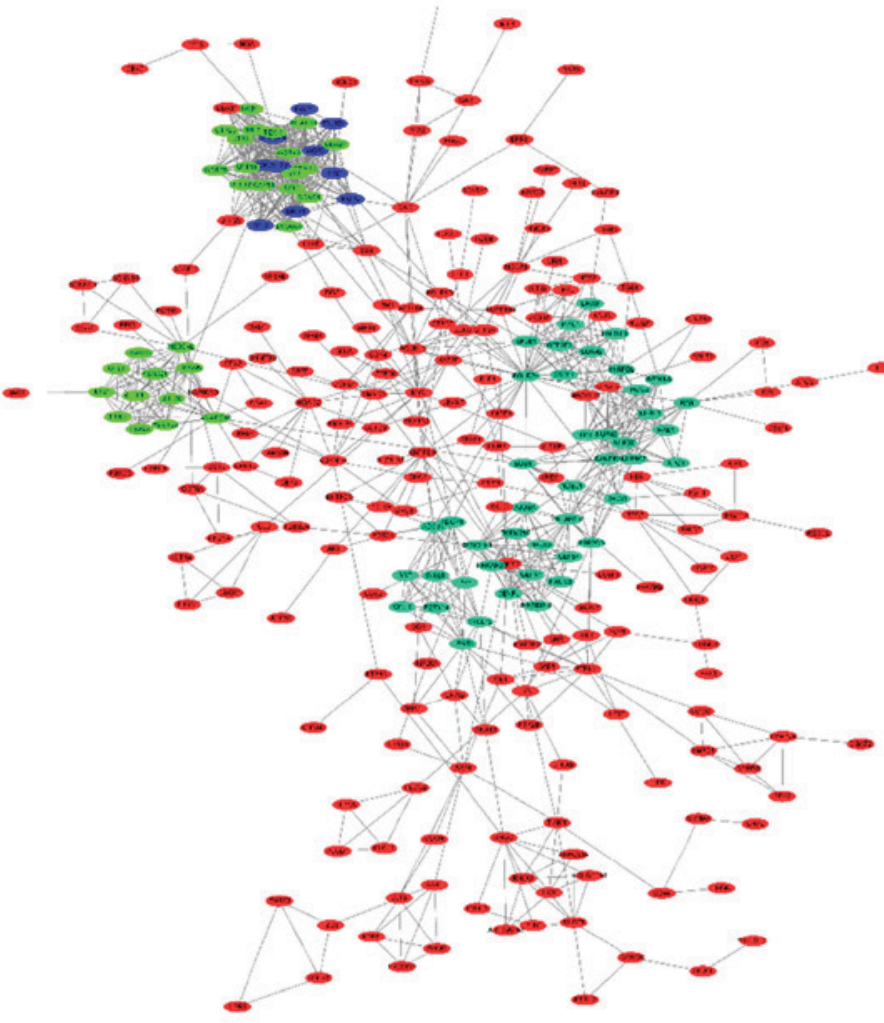

B

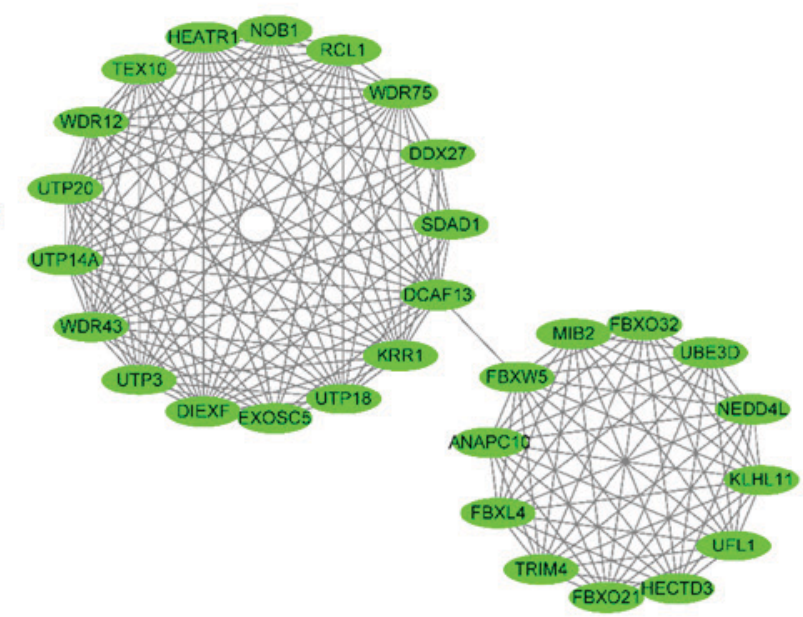

D

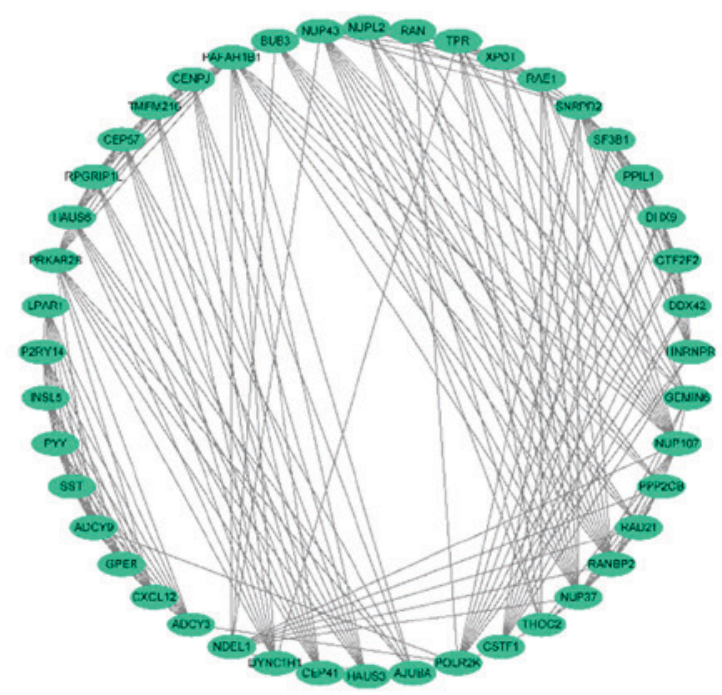

Figure 3. Interaction network of identified genes. (A) String tools were used to analyze protein-protein interactions between identified genes. A total of 306 genes were identified to be associated with a large complex interaction network. The weighted index of interactions were calculated, and three subgroups (separately represented in blue, green and light blue circles) with the highest inner correlations were identified. (B-D) Genes of each subgroup are presented in circular formations, in which interactions between genes are represented by gray lines.

(GSE28000, GSE21815, GSE44076 and GSE75970) (33-36) and performed GO enrichment analysis, KEGG pathway analysis and PPI network analysis. Guo et al (32) found also that cell cycle term may serve an important role in CRC; 31 hub genes were acquired in a PPI network. However, there was no intersection between these 31 hub genes and the results of the present study. A total of 17 pairs of cancer tissues and adjacent tissues were analyzed in the present study, which made obtained DEGs more reliable and accurate relative to unpaired samples. The follow-up analyses, including GO enrichment analysis, KEGG pathway enrichment analysis, PPI network analysis and MCODE software analysis, could be more reliable based on these DEGs. In addition, CRC tissues and adjacent tissues from patients with CRC were collected, and RT-qPCR was performed to verify the expression levels of the identified 20 hub genes in CRC tissue compared with normal tissue.

In the present study,DEGs in CRC and adjacent tissues were identified and were separated into two subgroups (upregulated and downregulated groups), but the accounts of DEGs are still enormous. Furthermore, the present study aimed to construct a protein interaction network based on the selected genes, and following this, a total of 306 genes were revealed to be involved in this network. From these 306 genes, 20 hub genes, which represent genes exhibiting close interactions with each other, were identified using the Cytohubba plug-in analysis. These hub genes may serve important molecular roles in the pathogenesis of CRC. 
Table II. List of 20 hub genes associated with colorectal cancer.

\begin{tabular}{rll}
\hline Rank & Gene & Location \\
\hline 1 & WDR3 & $1 \mathrm{p} 12$ \\
2 & WDR43 & $2 \mathrm{p} 23.2$ \\
3 & UTP14A & Xq26.1 \\
4 & DCAF13 & $8 \mathrm{q} 22.3$ \\
5 & KRR1 & $12 \mathrm{q} 21.2$ \\
6 & DIEXF & $1 \mathrm{q} 32.2$ \\
7 & HEATR1 & $1 \mathrm{q} 43$ \\
8 & WDR75 & $2 \mathrm{q} 32.2$ \\
9 & UTP18 & $17 \mathrm{q} 21.33$ \\
10 & UTP3 & $4 \mathrm{q} 13.3$ \\
11 & RCL1 & $9 \mathrm{p} 24.1$ \\
12 & UTP20 & $12 \mathrm{q} 23.2$ \\
13 & TEX10 & $9 \mathrm{q} 31.1$ \\
14 & WDR12 & $2 \mathrm{q} 33.2$ \\
15 & EXOSC5 & $19 \mathrm{q} 13.2$ \\
16 & NOB1 & $16 \mathrm{q} 22.1$ \\
17 & SDAD1 & $4 \mathrm{q} 21.1$ \\
18 & DDX27 & $20 \mathrm{q} 13.13$ \\
19 & RPF2 & $6 \mathrm{q} 21$ \\
20 & GTPBP4 & $10 \mathrm{p} 15.3$ \\
\hline
\end{tabular}

WDR3, WD repeat domain 3; WDR43, WD repeat domain 43; UTP14A, UTP14 small subunit processome component; DCAF13, damage specific DNA binding protein 1 and cullin4 associated factor 13; KRR1, KRR1 small subunit processome component; DIEXF, digestive organ expansion factor; HEATR1, HEAT repeat containing 1; WDR75, WD repeat domain 75; UTP18, UTP 18 small subunit processome component; UTP3, RCL1, RNA terminal phosphate cyclase-like 1; UTP20, UTP20 small subunit processome component; TEX10, testis expressed 10; WDR12, WD repeat domain 12; EXOSC5, exosome component 5; NOB1, neutral invertase (regulatory particle non-ATPase 12) binding protein 1; SDAD1, SDA1 domain containing 1; DDX27, DEAD-box helicase 27; RPF2, ribosome production factor 2; GTPBP4, GTP binding protein 4 .

GO analysis of the 20 hub genes revealed that there are four subunit processome component encoded genes: UTP3, UTP14A, UTP18 and UTP20 differentially expressed in CRC. The subunit processome component is an essential part of the ribonucleoprotein complex that once bound to the U3 small nuclear RNA, participates in ribosome biogenesis and $18 \mathrm{~S}$ ribosomal RNA synthesis (37). It has been demonstrated that alternative splicing of said genes may result in multiple transcript variants; which can promote cancer development (38). For example, UTP18 has been revealed to be localized in the cytoplasm of cells, and serum withdrawal has been revealed to increase cytoplasmic UTP18, which can associate with the translation complex and Hsp90 to upregulate the translation of HIF1a, Myc and VEGF, thus inducing a cellular stress response (39). UTP18 overexpression promotes transformation and tumorigenesis; however, UTP18 knockdown can inhibit these processes (23). In addition, the subunit processome component is represented by a large gene family (uridine triphosphate, UTP), which exhibits different mechanisms in

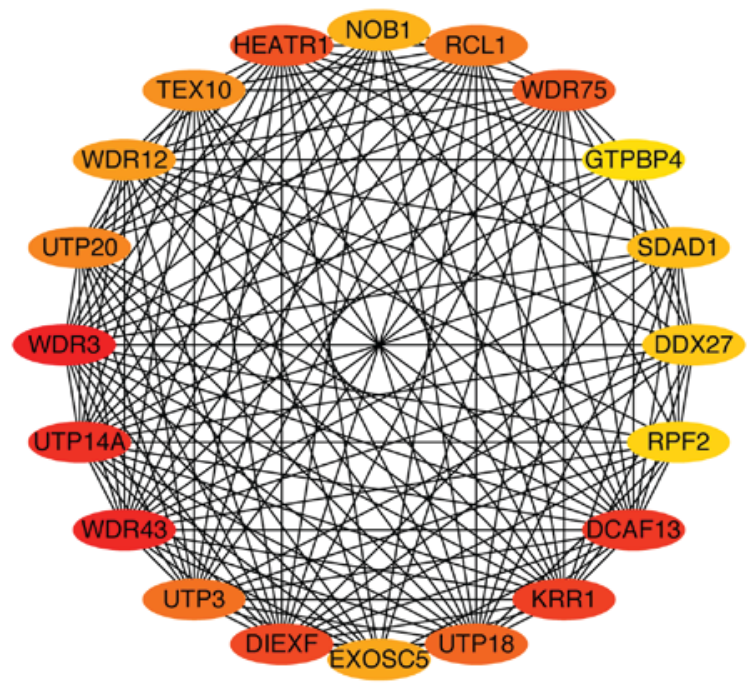

Figure 4. Interaction network of 20 hub genes. A total 20 hub genes were identified from 306 genes via MCC algorithm analysis. Each of the hub genes are presented as circle box linked each other by gray lines, presented as a PPI network. The color depth (yellow and light orange, medium orange and dark orange) of the boxes represents the degree of increasing importance in PPI network. PPI, protein-protein interaction.

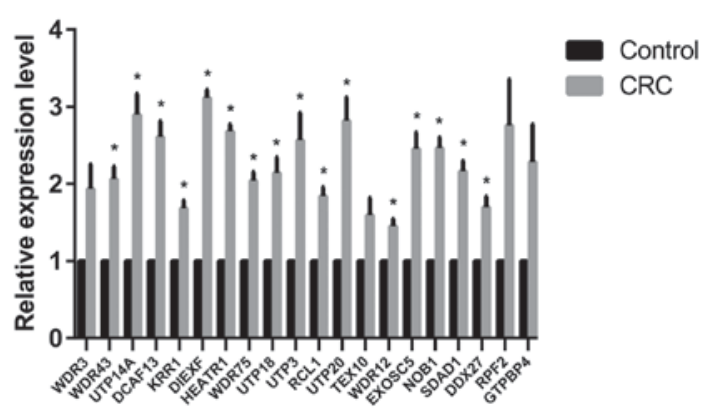

Figure 5. Reverse transcription-quantitative polymerase chain reaction analysis of the expression of 20 hub genes. Results were derived from three pairs of samples (paracancerous tissues and cancerous tissues). The expression levels of the 20 hub genes were significantly upregulated in CRC tissues relative to control tissues, consistent with the results of the microarray data analyses. ${ }^{*} \mathrm{P}<0.05$ vs. control.

cell proliferation and cancer pathogenesis (40). Furthermore, the associations between the four subunit processome component encoded genes and CRC, to the best of our knowledge, have not previously been investigated. The present study demonstrated that the aforementioned four genes may have important roles in the progression of CRC and represent potential biomarkers for CRC.

In the present study, members of the WD repeat domain family (including WDR3, WDR12, WDR43, WDR75) was a further group of genes revealed to be represented in the 20 hub genes. It has been previously established that WD repeats are 30-40 amino acid domains in length, and contain conserved repeating units, which are frequently terminated with Trp-Asp at the C-terminal (41). Proteins belonging to the WD repeat family are involved in numerous cellular processes, such as cell proliferation, apoptosis, signal transduction, gene regulation and human disease (42). In a recent study, Izumi et al (43) concluded that c-Myc expression alterations are regulated by upregulation of F-box/WD repeat-containing protein 7 (FBXW7), and that 
knockdown of FBXW7 via application of small interfering RNA could enhance cell sensitivity to anticancer agents.

Akdi et al (44) revealed that mRNA and protein levels of WDR3 are dysregulated in human thyroid cancer cells. In addition, a further study demonstrated that WDR3 can regulate genome stability in patients with thyroid cancer (45). However, studies investigating the associations between WD repeats and CRC are currently very limited. In the present study, it was revealed that these WD repeats are hub genes in progression of $\mathrm{CRC}$. Therefore, further in vitro and in vivo studies on this topic are required. He et al (46) demonstrated that knockdown of NOB1 can induce apoptosis of human colorectal cells. In addition, Zeng et al (47) demonstrated that knockdown of SDAD1 can suppress the proliferation, migration and invasion of colon cancer cells (47). The results of the previous two studies are consistent with the results of the present study. In addition, a previous study demonstrated that overexpression of DCAF13 in hepatocellular carcinoma is correlated with poor survival of patients (48). Dyachenko et al (49) demonstrated that KRR1 may represent a potential biomarker of particular histological types (invasive ductal) of breast tumor. Furthermore, Liu et al (50) revealed that HEATR1 can negatively regulate protein kinase B and further decrease resistance to gemcitabine and other chemotherapeutics. Tsukamoto et al (51) demonstrated that DDX27 is upregulated in gastric cancer tissues and may represent a potential therapeutic target for patients with gastric cancer. In addition, Liu et al (52) revealed that GTPBP4 serves an important role in hepatocellular carcinoma development, and that increased GTPBP4 expression is correlated with poor survival of patients with hepatocellular carcinoma. However, the functions of aforementioned five genes (DCAF13, KRR1, HEATR1, DDX27 and GTPBP4) have, to the best of our knowledge, not been investigated with regards to $\mathrm{CRC}$. The results of the present study demonstrated that the aforementioned five genes and the remaining 15 genes in the top 20 hub genes (DIEXF, RCL1, TEX10, EXOSC5 and RPF2) may serve important roles in CRC development and represent potential biomarkers for CRC.

In conclusion, the present study identified significant genes associated with the pathogenesis of CRC via analysis of genome-wide expression profiles of CRC as well as comparison of expression levels of significant genes in CRC tissues compared with healthy adjacent tissue samples. Furthermore, 20 hub genes were revealed via genetic analysis as validated by RT-qPCR. GO analysis revealed that 'small subunit processsome component' and 'WD repeat domains' were two protein family subgroups encoded by the 20 hub genes, and could represent novel molecular markers associated with CRC. The patterns of the expression levels of the 20 hub genes in CRC were further verified by RT-qPCR. However, a small sample size (three paired samples) represents a limitation of the present study, and therefore this should be further investigated using a greater sample size in future studies. Therefore, additional studies are required to further investigate the associations between identified hub genes and CRC.

\section{Acknowledgements}

We would like to express our thanks to the National Library of Medicine (https://www.nlm.nih.gov/) for giving user the privilege to freely download the raw data of various GEO series.

\section{Funding}

No funding was received.

\section{Availability of data and materials}

The datasets used and analyzed during the current study are available from GEO database (GSE32323, https://www.ncbi. nlm.nih.gov/geo/query/acc.cgi?acc=GSE32323).

\section{Authors' contributions}

All the research was conducted by SL. The design of the present study was conceived by QH. All authors read and approved the final manuscript.

\section{Ethics approval and consent to participate}

Written informed consent was obtained from all patients included in the present study. The Ethics Committee of Renji Hospital Affiliated to Shanghai Jiaotong University School of Medicine approved the present study and protocols.

\section{Consent for publication}

Written informed consent was obtained from all patients for publication of reverse transcription-quantitative polymerase chain reaction results in this study; personal identifying information was not included in this article.

\section{Competing interests}

The authors declare that they have no competing interests.

\section{References}

1. McWhirter JE and Hoffman-Goetz L: Coverage of skin cancer and recreational tanning in North American magazines before and after the landmark 2006 international agency for research on cancer report. BMC Public Health 15: 169, 2015.

2. Navarro M, Nicolas A, Ferrandez A and Lanas A: Colorectal cancer population screening programs worldwide in 2016: An update. World J Gastroenterol 23: 3632-3642, 2017.

3. Marley AR and Nan H: Epidemiology of colorectal cancer. Int J Mol Epidemiol Genet 7: 105-114, 2016.

4. Siegel RL, Miller KD and Jemal A: Cancer statistics, 2016. CA Cancer J Clin 66: 7-30, 2016.

5. Ranzani M, Annunziato S, Adams DJ and Montini E: Cancer gene discovery: Exploiting insertional mutagenesis. Mol Cancer Res 11: 1141-1158, 2013

6. Li MH, Fu SB and Xiao HS: Genome-wide analysis of microRNA and mRNA expression signatures in cancer. Acta Pharmacol Sin 36: 1200-1211, 2015.

7. Choi JY, Kim JG, Lee YJ, Chae YS, Sohn SK, Moon JH, Kang BW, Jung MK, Jeon SW, Park JS and Choi GS: Prognostic impact of polymorphisms in the CASPASE genes on survival of patients with colorectal cancer. Cancer Res Treat 44: 32-36, 2012.

8. Lascorz J, Chen B, Hemminki K and Försti A: Consensus pathways implicated in prognosis of colorectal cancer identified through systematic enrichment analysis of gene expression profiling studies. PLoS One 6: e18867, 2011.

9. Karagiannis GS, Berk A, Dimitromanolakis A and Diamandis EP: Enrichment map profiling of the cancer invasion front suggests regulation of colorectal cancer progression by the bone morphogenetic protein antagonist, gremlin-1. Mol Oncol 7: 826-839, 2013. 
10. Lascorz J, Hemminki K and Försti A: Systematic enrichment analysis of gene expression profiling studies identifies consensus pathways implicated in colorectal cancer development. J Carcinog 10: 7, 2011.

11. Khamas A, Ishikawa T, Shimokawa K, Mogushi K, Iida S, Ishiguro M, Mizushima $\mathrm{H}$, Tanaka $\mathrm{H}$, Uetake $\mathrm{H}$ and Sugihara $\mathrm{K}$ Screening for epigenetically masked genes in colorectal cancer Using 5-Aza-2'-deoxycytidine, microarray and gene expression profile. Cancer Genomics Proteomics 9: 67-75, 2012.

12. Irizarry RA, Hobbs B, Collin F, Beazer-Barclay YD, Antonellis KJ, Scherf U and Speed TP: Exploration, normalization, and summaries of high density oligonucleotide array probe level data. Biostatistics 4: 249-264, 2003.

13. Wu Z, Irizarry RA, Gentleman R, Martinez-Murillo F and Spencer F: A model-based background adjustment for oligonucleotide expression arrays. J Am Stat Assoc 99: 909-917, 2004

14. Denoeux T: A k-nearest neighbor classification rule based on Dempster-Shafer theory. Syst Man Cybernetics IEEE Trans 25 804-813, 1995

15. Smyth GK: Limma: Linear models for microarray data Bioinformatics Comput Biol Solutions Using R Bioconductor: 397-420, 2011.

16. Maere S, Heymans K and Kuiper M: BiNGO: A Cytoscape plugin to assess overrepresentation of gene ontology categories in biological networks. Bioinformatics 21: 3448-3449, 2005.

17. Jiao X, Sherman BT, Huang da W, Stephens R, Baseler MW, Lane HC and Lempicki RA: DAVID-WS: A stateful web service to facilitate gene/protein list analysis. Bioinformatics 28 : 1805-1806, 2012

18. Kanehisa M and Goto S: KEGG: Kyoto encyclopedia of genes and genomes. Nucleic Acids Res 28: 27-30, 2000.

19. Livak KJ and Schmittgen TD: Analysis of relative gene expression data using real-time quantitative PCR and the 2(-Delta Delta C(T)) method. Methods 25: 402-408, 2001.

20. Huang D, Sun W, Zhou Y, Li P, Chen F, Chen H, Xia D, Xu E, Lai M, Wu Y and Zhang $\mathrm{H}$ : Mutations of key driver genes in colorectal cancer progression and metastasis. Cancer Metastasis Rev 37: 173-187, 2018.

21. Janin N: A simple model for carcinogenesis of colorectal cancers with microsatellite instability. Adv Cancer Res 77: 189-221, 2000.

22. Yagdi Efe E, Mazumder A, Lee JY, Gaigneaux A, Radogna F, Nasim MJ, Christov C, Jacob C, Kim KW, Dicato M, et al: Tubulin-binding anticancer polysulfides induce cell death via mitotic arrest and autophagic interference in colorectal cancer. Cancer Lett 410: 139-157, 2017.

23. Otto T and Sicinski P: Cell cycle proteins as promising targets in cancer therapy. Nat Rev Cancer 17: 93-115, 2017.

24. Liu Z, Li J, Chen J, Shan Q, Dai H, Xie H, Zhou L, Xu X and Zheng S: MCM family in HCC: MCM6 indicates adverse tumor features and poor outcomes and promotes $\mathrm{S} / \mathrm{G} 2$ cell cycle progression. BMC Cancer 18: 200, 2018.

25. Owa T, Yoshino H, Yoshimatsu K and Nagasu T: Cell cycle regulation in the G1 phase: A promising target for the development of new chemotherapeutic anticancer agents. Curr Med Chem 8: $1487-1503,2001$

26. Roberge M, Berlinck RG, Xu L, Anderson HJ, Lim LY, Curman D, Stringer CM, Friend SH, Davies P, Vincent I, et al: High-throughput assay for G2 checkpoint inhibitors and identification of the structurally novel compound isogranulatimide. Cancer Res 58: 5701-5706, 1998.

27. Merlo A, Mabry M, Gabrielson E, Vollmer R, Baylin SB and Sidransky D: Frequent microsatellite instability in primary small cell lung cancer. Cancer Res 54: 2098-2101, 1994.

28. Tashiro K, Iwamuro S, Hatano T, Furuta A, Takizawa A, Ohishi Y, Igarashi H, Hasegawa N, Asano K and Aoki H: Double cancer observed from bladder cancer. Nihon Hinyokika Gakkai Zasshi 90: 509-513, 1999.

29. Di Franco S, Turdo A, Todaro M and Stassi G: Role of type I and II interferons in colorectal cancer and melanoma. Front Immunol 8: 878, 2017.

30. Song D, Huang R, Tang Q, et al: Identification of EZH2-related key pathways and genes in colorectal cancer using bioinformatics analysis. Chin J Colorectal Dis 5: 475-479, 2016

31. Liang B, Li C and Zhao J: Identification of key pathways and genes in colorectal cancer using bioinformatics analysis. Med Oncol 33: 111, 2016.

32. Guo Y, Bao Y, Ma M and Yang W: Identification of key candidate genes and pathways in colorectal cancer by integrated bioinformatical analysis. Int J Mol Sci 18: pii:E722, 2017.
33. Sanz-Pamplona R, Berenguer A, Cordero D, Molleví DG, Crous-Bou M, Sole X, Paré-Brunet L, Guino E, Salazar R, Santos C, et al: Aberrant gene expression in mucosa adjacent to tumor reveals a molecular crosstalk in colon cancer. Mol Cancer 13: 46, 2014.

34. Kogo R, Shimamura T, Mimori K, Kawahara K, Imoto S, Sudo T, Tanaka F, Shibata K, Suzuki A, Komune S, et al: Long noncoding RNA HOTAIR regulates polycomb-dependent chromatin modification and is associated with poor prognosis in colorectal cancers. Cancer Res 71: 6320-6326, 2011.

35. Jovov B, Araujo-Perez F, Sigel CS, Stratford JK, McCoy AN, Yeh JJ and Keku T: Differential gene expression between African American and European American colorectal cancer patients. PLoS One 7: e30168, 2012.

36. Huang FT, Chen WY, Gu ZQ, Zhuang YY, Li CQ, Wang LY, Peng JF, Zhu Z, Luo X, Li YH, et al: The novel long intergenic noncoding RNA UCC promotes colorectal cancer progression by sponging miR-143. Cell Death Dis 8: e2778, 2017.

37. Melnik S, Deng B, Papantonis A, Baboo S, Carr IM and Cook PR: The proteomes of transcription factories containing RNA polymerases I, II or III. Nat Methods 8: 963-968, 2011.

38. Narla A and Ebert BL: Ribosomopathies: Human disorders of ribosome dysfunction. Blood 115: 3196-3205, 2010.

39. Yang HW, Kim TM, Song SS, Menon L, Jiang X, Huang W, Black PM, Park PJ, Carroll RS and Johnson MD: A small subunit processome protein promotes cancer by altering translation. Oncogene 34: 4471-4481, 2015.

40. Guan Y, Huang D, Chen F, Gao C, Tao T, Shi H, Zhao S, Liao Z, Lo LJ, Wang Y, et al: Phosphorylation of Def regulates Nucleolar p53 turnover and cell cycle progression through Def recruitment of Calpain3. PLoS Biol 14: e1002555, 2016.

41. Neer EJ, Schmidt CJ, Nambudripad R and Smith TF: The ancient regulatory-protein family of WD-repeat proteins. Nature 371: 297-300, 1994.

42. Li D and Roberts R: WD-repeat proteins: Structure characteristics, biological function, and their involvement in human diseases. Cell Mol Life Sci 58: 2085-2097, 2001.

43. Izumi D, Ishimoto T, Miyake K, Eto T, Arima K, Kiyozumi Y, Uchihara T, Kurashige J, Iwatsuki M, Baba Y, et al: Colorectal cancer stem cells acquire chemoresistance through the upregulation of F-Box/WD repeat-containing protein 7 and the consequent degradation of c-Myc. Stem Cells 35: 2027-2036, 2017.

44. Akdi A, Giménez EM, Garcia-Quispes W, Pastor S, Castell J, Biarnés J, Marcos R and Velázquez A: WDR3 gene haplotype is associated with thyroid cancer risk in a Spanish population. Thyroid 20: 803-809, 2010.

45. Garcia-Quispes WA, Pastor S, Galofré P, Biarnés J, Castell J, Velázquez A and Marcos R: Possible role of the WDR3 gene on genome stability in thyroid cancer patients. PLoS One 7: e44288, 2012.

46. He XW, Feng T, Yin QL, Jian YW and Liu T: NOB1 is essential for the survival of RKO colorectal cancer cells. World J Gastroenterol 21: 868-877, 2015.

47. Zeng M, Zhu L, Li L and Kang C: miR-378 suppresses the proliferation, migration and invasion of colon cancer cells by inhibiting SDAD1. Cell Mol Biol Lett 22: 12, 2017.

48. Cao J, Hou P, Chen J, Wang P, Wang W, Liu W, Liu C and He X: The overexpression and prognostic role of DCAF13 in hepatocellular carcinoma. Tumour Biol 39: 1010428317705753, 2017.

49. Dyachenko L, Havrysh K, Lytovchenko A, Dosenko I, Antoniuk S, Filonenko V and Kiyamova R: Autoantibody response to ZRF1 and KRR1 SEREX antigens in patients with breast tumors of different histological types and grades. Dis Markers 2016: 5128720, 2016.

50. Liu T, Fang Y, Zhang H, Deng M, Gao B, Niu N, Yu J, Lee S, Kim J, Qin B, et al: HEATR1 negatively regulates Akt to help sensitize pancreatic cancer cells to chemotherapy. Cancer Res 76: 572-581, 2016.

51. Tsukamoto Y, Fumoto S, Noguchi T, Yanagihara K, Hirashita Y, Nakada C, Hijiya N, Uchida T, Matsuura K, Hamanaka R, et al: Expression of DDX27 contributes to colony-forming ability of gastric cancer cells and correlates with poor prognosis in gastric cancer. Am J Cancer Res 5: 2998-3014, 2015.

52. Liu WB, Jia WD, Ma JL, Xu GL, Zhou HC, Peng Y and Wang W: Knockdown of GTPBP4 inhibits cell growth and survival in human hepatocellular carcinoma and its prognostic significance. Oncotarget 8: 93984-93997, 2017.

This work is licensed under a Creative Commons Attribution-NonCommercial-NoDerivatives 4.0 International (CC BY-NC-ND 4.0) License. 